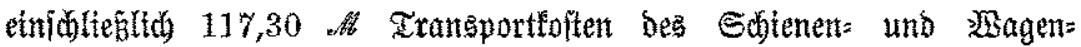
materials vom Magazin an die Sebraudeflelle. Die Sinzeltoften der Şerittellung ohne biejelben mit 787,11 Al waren jummarifich für

Flanieren und Syeritellung des Fangoammes . . . 141,96 M

Majolinenteile mit Rollen . . . . . . . . . 189,84"

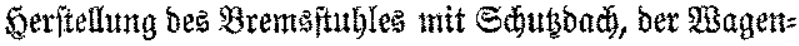

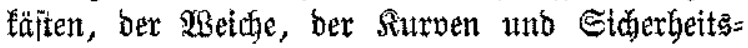

geländer, Montieren ber Majudinenteile . . . 250,02 "

Drebitibe . . . . . . . . . . . 54,46"

Drahtifeil. . . . . . . . . . . . 111,03" "

Iufwifelrolle bietzu . . . . . . . . . . . 21,50"

Marnungstafel . . . . . . . . . . . . . 5,00"

Fette zur fonjernterung . . . . . . . $\frac{.}{\text { Sa. } 787,11 \text { / } 13 \text { w. o. }}$

马m Betriebjabre 1900 wurón $497 \mathrm{rm}$ Stente ouf bem Brems= berge geẗbrbert und wurbe hierbet gegenitber bem ânfaut non Steinett eine Eriparang von rund $900 \mathscr{A b}$ erzielt, fo bas bie STerftellung: foiten ber Anlage kereits im erften Betriebsjabre amortifiert waren. Rünftig fann bex Raummeter Steine um 1,80 M billiger gelief̧ert werben als vor Anlage bes sBremeberges.

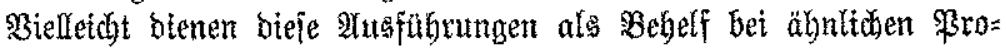

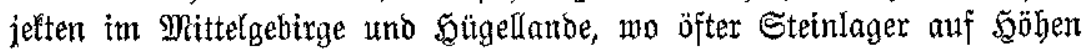

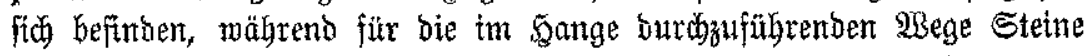
ourtftity nicht vorbanden find.

Reuenịorg (Dberfanfen), im Thá 1901.

S. Piantel, figl. Foritamteijeffor.

\title{
II. Mttitteilungen.
}

II. Gqauptwerfammlung des deutichen forftereins (29. Der: fammlung deutfiher forftmänner) in Regensburg.

1. Bevioft îber bie Sthungen.

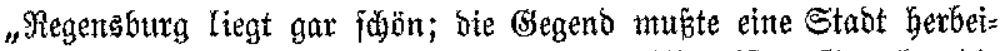
loden." Mit btejen Sorten rühmt idon ber Mttmeifter Goethe bie

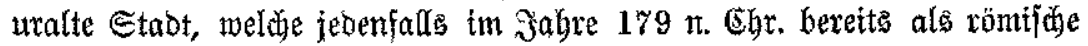
Rieberlafjung beitanben und jeitbem in ber Gefidite Deutiblands eire bobe \$ebeutung erlangt bat. Sein Mutnder baker, bap befien 


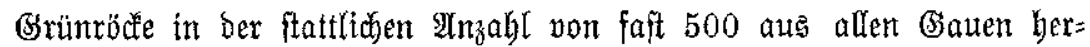
beiftrömten, um in bem intereffanten, altebrmüroigen Drte für einige

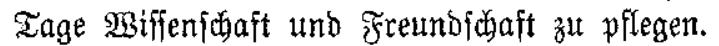

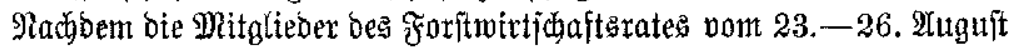
Borberatungen gepflogen batten, eröffnete Dberforitmeifter $\mathfrak{M}$ a am

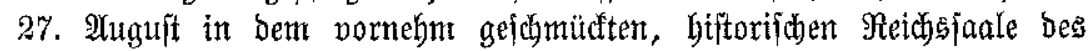

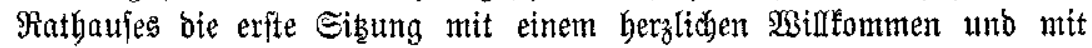

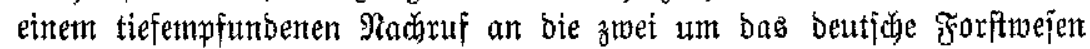

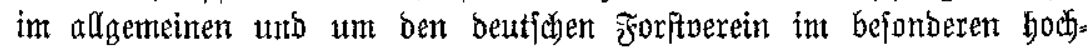
verbienten Männer aus Sorb und Süb: Dandelmann unb Bang= hofer. Es folgten freundlide 3 egrüpungen ber Berjammlung feitens Des Ehefs der bayerifben Foritnermaltung, Minifterialrat v. Sat ber,

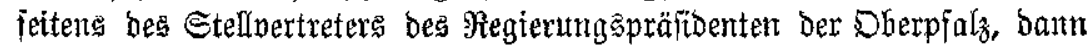
bes Bürgermetifters dex gaftlicten Stabt Regensburg, auth feitens bes

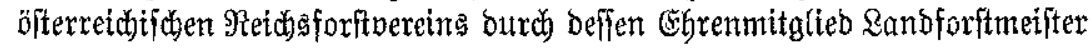

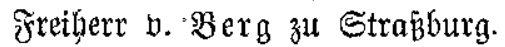

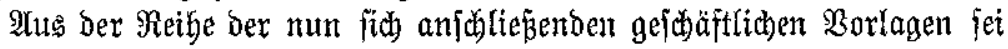

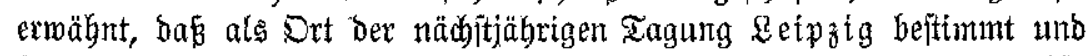

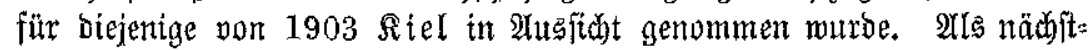
jährige Sauptthemata follen behandelt werben: 1. im Şinblicf auf bas Reipziger Exturitonsebiet: "Die llmwandung geringer Mittelwalbbeftände in Mabel'holz uno Deren Erfolge," 2. "Darlegutng ber SGrunojätse für bie Beleihung von Waldungen."

Şierauf wurbe tas erite Thema erörtert:

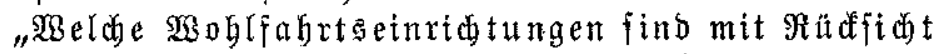
auf ben beftehenden atrbeiter= Mangel für bie $\mathfrak{M a l b}$ arbeiterida a t $z^{\prime}$ trefjen?"

Berichteritatter, Gebeimer Kammerrat at nónberg=Braumfineig, nahm bei seipredung Der Frage vorzugsmeife bie Ranbesbezinfe I bis V unb XIV bis XVI (bie nörolichen und noroöitlichen Bebiete Deut jo =

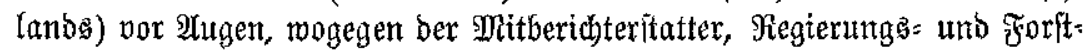

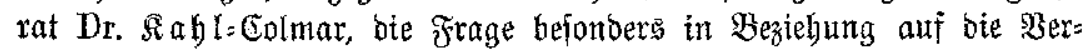
Gältniffe in ben übrigen Ranbesbezirfen (VI bis XIII) behandelte.

Beibe hatten 仿通 auf bie nadffolgenden Reitfäbe geeinigt:

I. Mangel an 2 trbeitsträften für ben Foritbetrieb, wie ihn die Frage

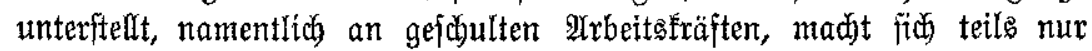
zeitmeije, teifs anbauerno, bereits auf grojen Gebieten füblbar. $\mathfrak{A} \mathfrak{n}$ vielen Drten wirb über Mangel entweder nur an tübtigen Sgolzhauern over an fultur= und $\mathfrak{B}$ egearbeitern geflagt, in mandjen Gebieten mit bidbter Bemaloung ftehen für Betriebsarbeiten jeder 2 trt unb zu jeber 


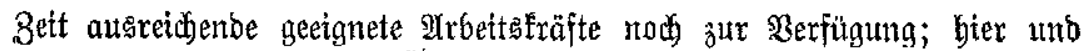

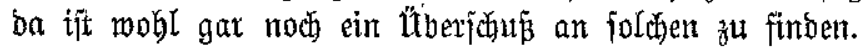

II. Die Mriachen bes auftretenden Mangels, namentlich an tüchitigen

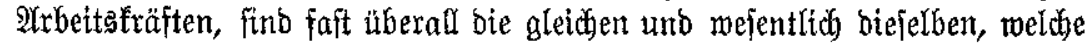
bie bedenfliche 2arbeiternot in ber Ranbwirtfobaft gezeitigt baben. (Die in ben neueften \$erioden bes allgemetnen wirtichaftlichen 2 tuffidnunges

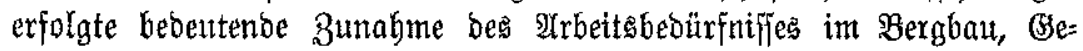
werbe, in ber Sndouftrie und dem Iransportmejen einerjeits, anbererfeits

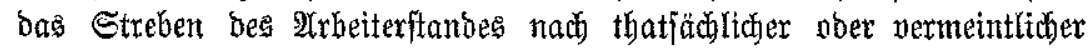

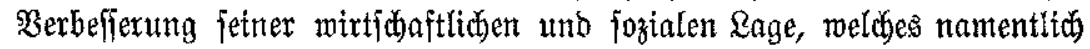
bie jüngeren Reute zux Abmantberung nach ben Stäoten und Snbuftrie= centren veranlafist.)

III. Die vorliegende Frage bejogränlft bie Berichterftatter auf bie

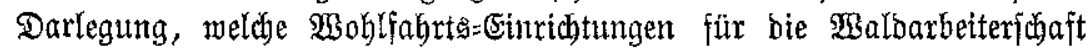
angezeigt und geeignet erijjeinen, bem bereits beftehenden ober fïr bie Folge zut bejorgenden $\mathfrak{A r b e i t e r m a n t g e l ~ m i t ~ d e n ~ b a r a u t s ~ f u ̈ r ~ b i e ~}$

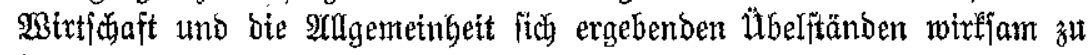
begegnen. Dieje Frage und ihre \&opipung berifgrt nidgt nur bie wirtichaft= lidhen Sntereffen ber Balbbefither uno ber Malbarbeiter, jondern wegen ber mannigfachen Beziebungen znifdhen ber Forit= uno Sanduirtichaft in gemiffem Grabe autch bte Snterefien ber Ieteteren; fie ift aber zugleith non allgemeiner jozialpolitifoter seoututng.

IV. 2In geeigneten Maß̧nabmen fommen in Setracht und finto, je

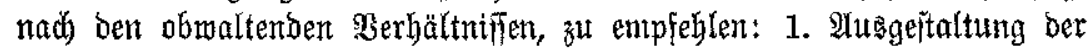
Irbeiternerficherung in Bsoztg auf Fürionge bet ßrantheitsfällen ber

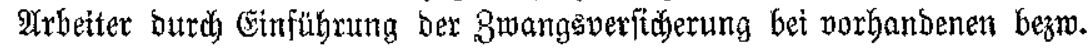
neut zut jofaffenben Srtbs=, Betriebs= ober gemeinjamen Bemeinbefranfen= kafien. 2. Fürrjorge für Unfallverbütung, lunterweijung in ber Şilfe=

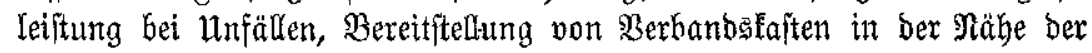

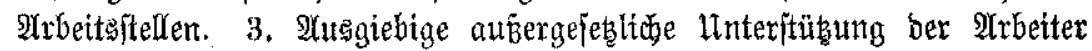

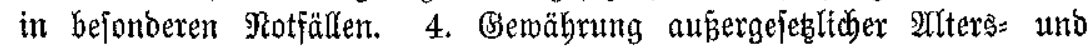

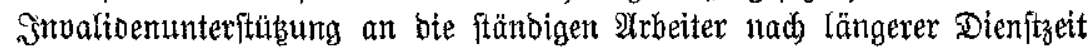

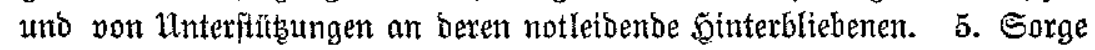

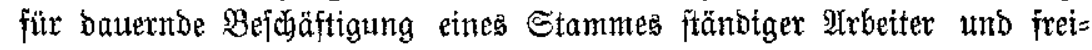

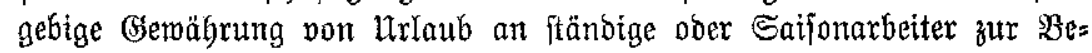

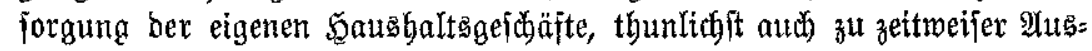
übung bejonbers Iohnender anberweitiger (Ermerbsthätigfelt. 6. Ber=

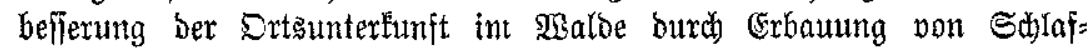
häujern unb geräumigen Schubghütten. \%. Bermittlıng bes 2 Infaufs gut

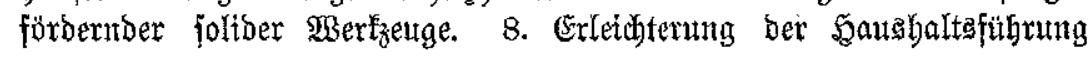


Der Arbeiter burch Ranbverpactung unb Gewährung von Saturalbezingen

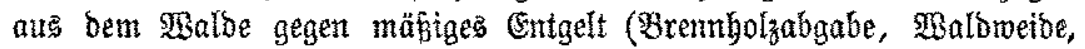
(6ras uno Geimftätten, entweber anf Roften Des \$alobefiners zu billiger miet=

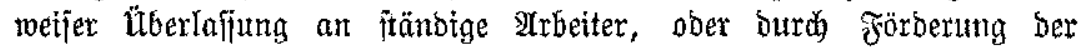

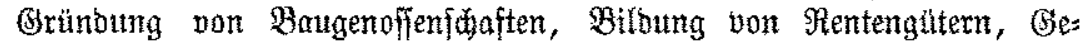

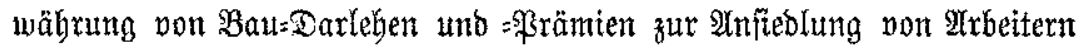
(Sialonifation). 10. Bilbung von fonfigen Bereinen uno Genoffenichaften

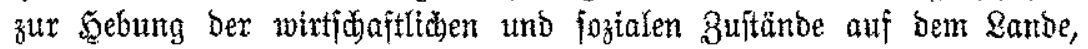
beza. Förberung foldjer Beftrebungen, als: Bilbung von Spar= und

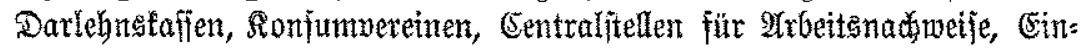

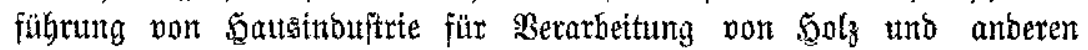

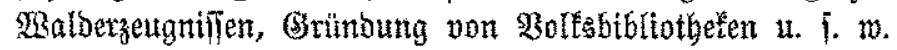

$\nabla$. Bieten Maßnabmen ber vorgebachtent 2 rrt, insbefondere bie $\mathfrak{B} e=$

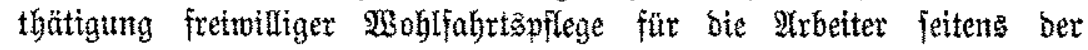
Urbeitgeber mb ifrer Drgane aud) bebentiante Mittel, "bie Mrbetter an ben Balb zu feffeln", fo befält gleitwohl bie Bewährung eines aus:

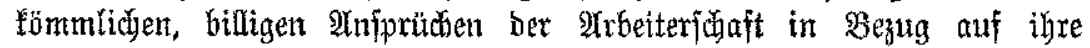
Rebenghaltung entfprechenden Befantlohnes hervorragenbe Bebeutung.

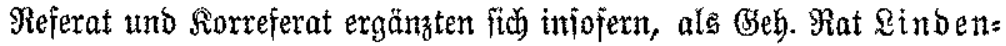
berg ben allgemeinen Tetl etwas eingehenber betanbelte unb bierbei bie Borfragen beantwortete: 1. ŞSt ein Mangel an 2 Irbeitsträften in ber

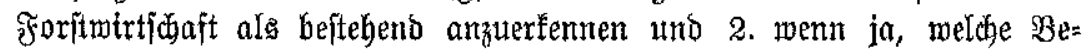
beutung ift ibm beizumeffen. Referent bejabt bie exite Frage, entmicfelt

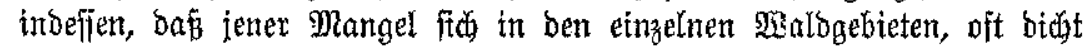

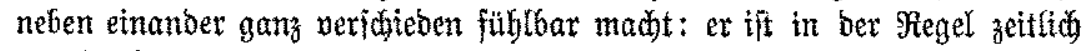

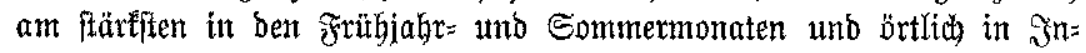
buftriecentren, in ber Mähe grober Stäbte uno an ber See. Bafl fehlt

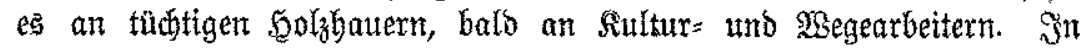

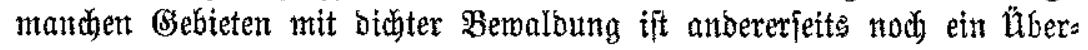

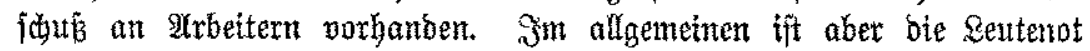
bei ber extenfineren Forftwirtifalt nod lange nibt fo faslimm, als bet

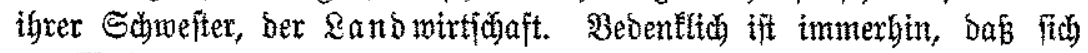

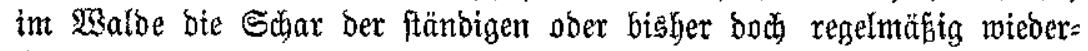

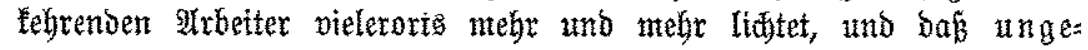

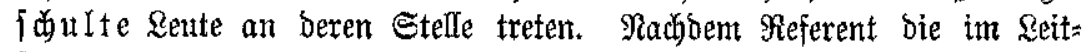
fab II bexübrten $\mathfrak{H}$ rifachen bes $\mathfrak{B a l b a r b e i t e r m a n g e l s ~ e i n g e b e n d e r ~ b e = ~}$ fprochen, legt ex bie zur $\mathscr{U}$ fbiffe geeigneten $\mathfrak{M a}$ âregeln im einzelnen bar; Gierbei nimant er namentlid auf bie in feinem Seimatlanbe \$raunionweig

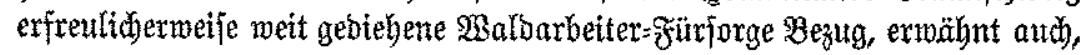




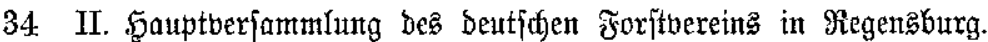

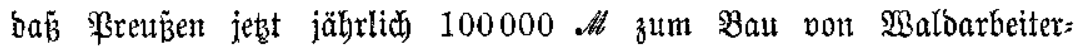

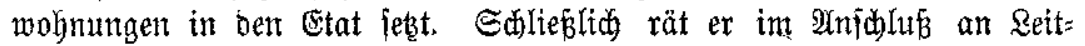
jak $V$, jebenfalls aud der 20 hnfrage bie gebithrenbe Beachtung zu wiomen;

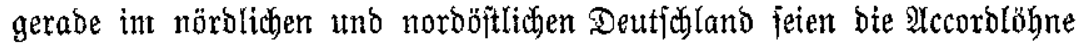

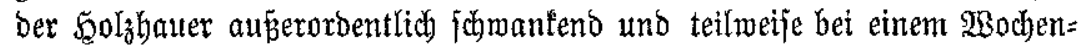

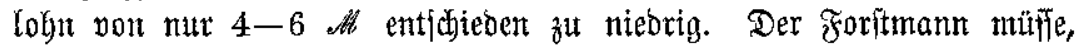
um ber 2 fbwanberung norzubeugen, an ber Sebung ber matertellen Rage wie aud ber Sittlichleit, jowie Des Gemüts= und Geifteslebens peiner 2Urveiter nacis Siäften mitwirkent.

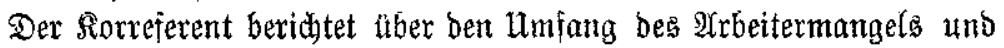
bie daburd hervorgerufenen Betriebsftörungent in Süben uno Meften

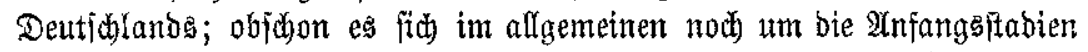
von Salbarbeiternot Gandelt, fo mïffe, namentlich nach ben lobentswerten Fürjorgema pregeln jeitens ber induftriellen Wrbeitgeber, immerhin weit

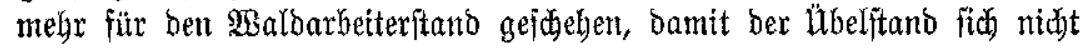

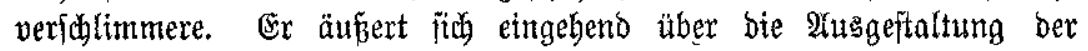
Srankenverfiberung ber Saldarbetter; viele berjelben entbehren leiber eitre joldhe nod) ganz. Wrs nadabmenawert merben hingejteflt bie fönigl. Gayerifhe, bie heffifo und einige preubifole Forftbetriebs= frantentaffen.

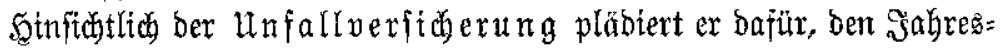

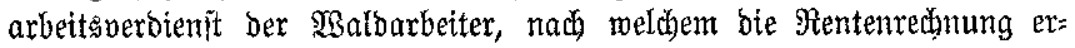
folgt, nid)t zu fnupp zu betneffen, namentlid) jenen nötigenfalls böber zu normieren, als ben ber lanbwirtidjaftlidgen 2 drbeiter.

Ex tritt ferner ein für bì Ergänz̧ung ber Reiftungen ber $\mathfrak{A r b e i t e r =}$

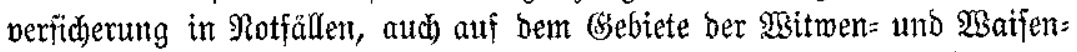

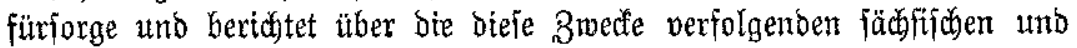

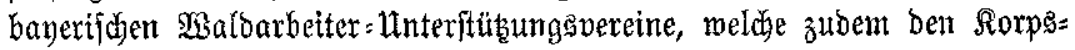
geift pflegen uno ben $\mathfrak{M}$ beiter an ben $\mathfrak{W a}$ ald fefjeln.

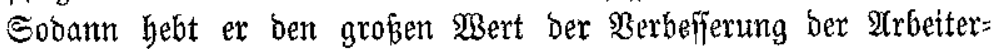

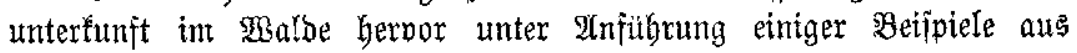
Eothringen und $\mathfrak{B a b e n , ~ m o ~ g u t e ~} 3$ aldberbergen entitanden finb.

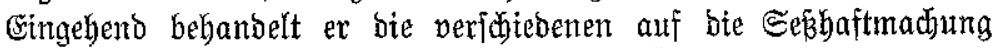

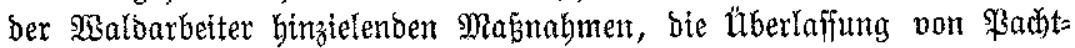

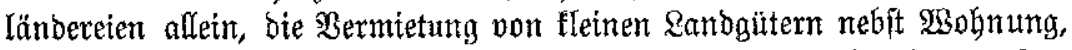

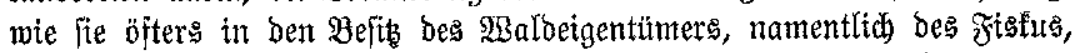

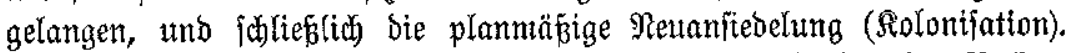

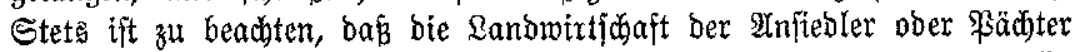

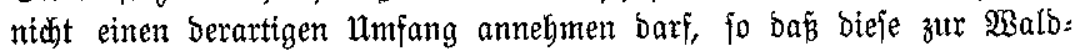
arbeit nidbt genügend Zeit übrig haben. SBis jebt beftehen betipielameije 


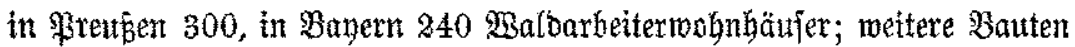

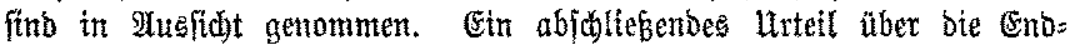

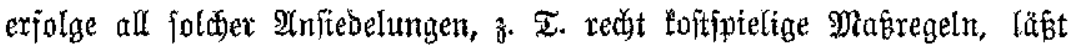
(ic) not nitht abgeben.

Bur zahnfrage bemerlt bex Sorreferent, bas nad ben allerbings

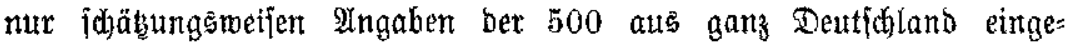

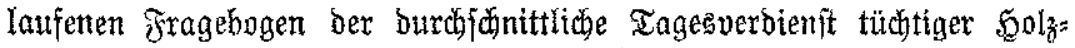

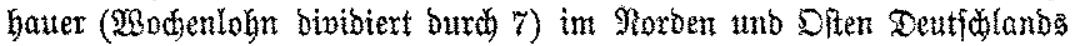

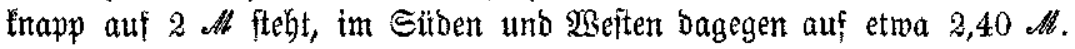

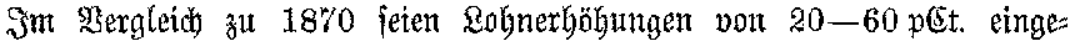
treten. (SIleidhwobl meroen hier und ba weitere Muffefferungen geboten jein, um bie $\mathfrak{A} r b e i t e r$ zu halten.

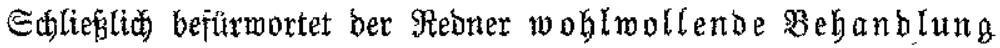
ber פalbarbetter und bie 2 us zei di nung foldher nad lanjähriger, treu geleifiteter Dienftzeit.

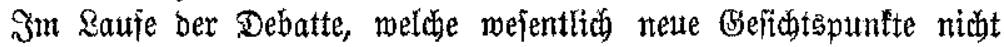

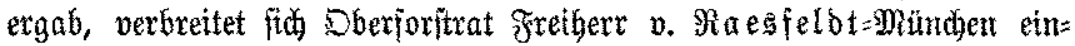
gebenoer über bie erfte Silfeleiftung bei setriebsunfällen; in Bayernt find zu biejent 3 wede auf einjamen Foritbäujern und it 2 rbeiters

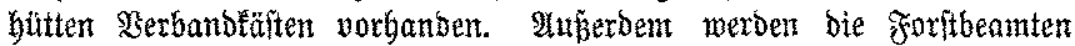
unb $\mathfrak{S}$ olzhauermeiftex im fogenannten Samariterbienit unterwielen; folde Surje werben aud auf ben פ̧albbaujchulen abgehalten.

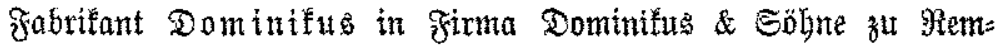

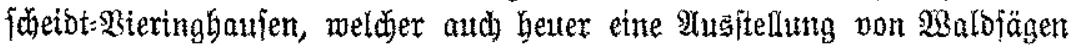

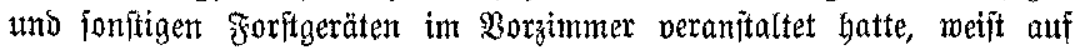
bie guten Erfahrungen mit feinen Fabrifaten hin; mit benfelben fönne

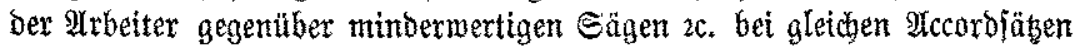

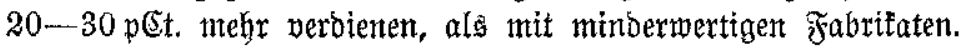

Dherforitnteifter Dr. Borggreve= BBieşbabest if allerbing bafur,

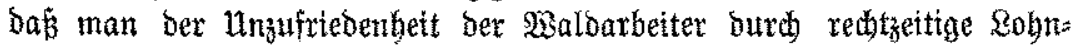

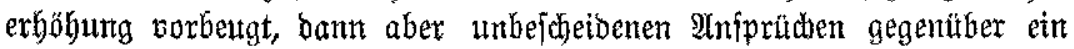

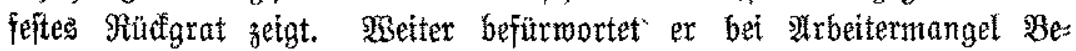
făräntung der Sommerarbett, mit weldem Borjhlage bie Bericht= erftatter f̈u im allgemeinen nicht einveritanden erffären; es jei vieffach gerabezu ein Bebürfnis, burdh Gewährung von Sommerarbeit, z. $\mathfrak{3}$.

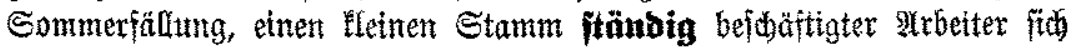
zu fichern.

Giermit wird bag Thema itber bie Maloarbeiterfrage verlaffen.

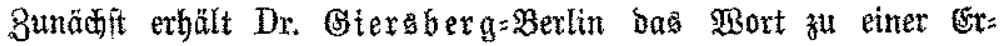
örterung Der Dïngungsverfude im $\mathfrak{W}$ ald e; Der Reoner, Merfaffer 


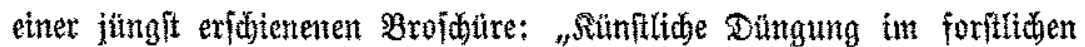
Betrebe" fpridit als Şertreter ber trei grofen beutichen Düngex:

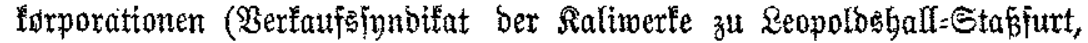

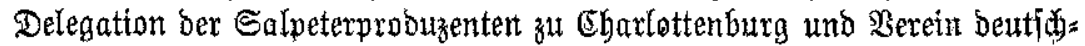
öfterreichijoder Ihomasphosphatfabrifen zu Berlin) vielleid)t etwas jebr "pro domo". Er befürmortet bringenb, nachbem bie fünftlicte Dünguttrg

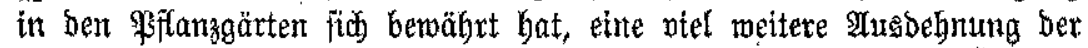
Serpude in Frreifulturen. Wsenn es gelungen ift, fitmmernbe boland:

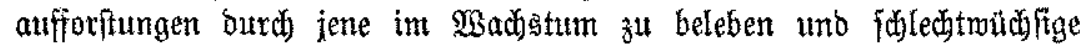
Budhenjungwübje ipeziell mittele Stictiftoffoüngutg anzuregen, fo jollten

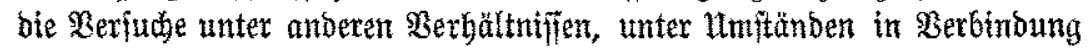
mit Grüntüngung ober Tieflockerung fortgejekst werden, zumal bie vot=

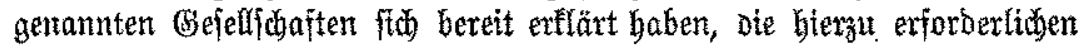

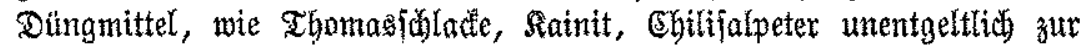
Berfügung zu ftellen.

Forftmeifter Dr. Sentf(c)= Müntsen hält Dte vom Borresner aufs geworfene Frage für widhtig unb aftuell; mit Der bisherigen Theorie "Der $\mathfrak{B a l}$ lo büngl fich felbit" müffe man um fo mebr brechen, als biefer

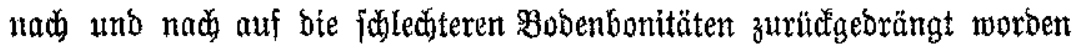

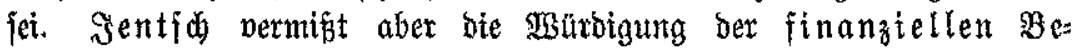
laftung in Bergletdi) zu ben zu erroartenoen (Exfolgen; ferner tritt ex für

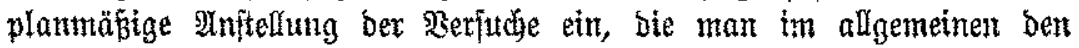
\$raftifern allein nidgt überlafjen bürfe.

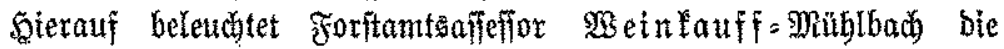
Düngungegfrage in Ridhte feiner neuen Symustheorie, beren Darlegumg in ber Corona mandes Sopflidjutteln berworruft. Sebner erflärt bie in Walbe fo oft wahrnebmbare "Booenfidminofutht" nidht als eine Folge von Waflermangel, vielmely als bie Wintung einex ungenugenben $3 \mathrm{er}=$ fetwing bes Fumus. Ex betradtet folden auf bem Boben nie als

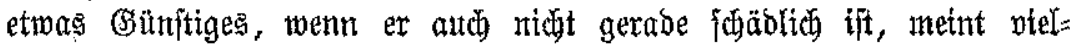

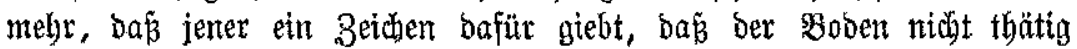

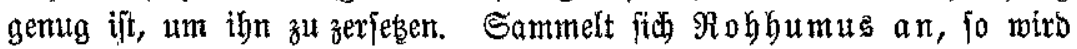

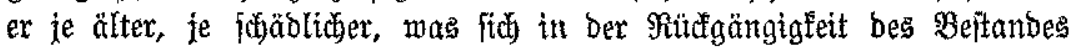
äuneert. Daher foll man ber fiefer, weil fie bie Rohbumusbiloung förbert, thunlichit bie \$odje ober bie Tanne beigeben, uberbaupt ge:

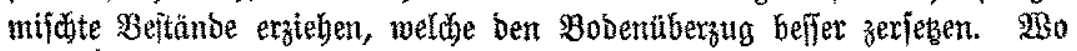

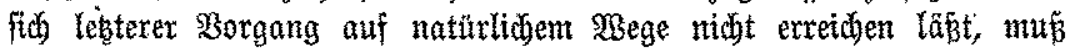

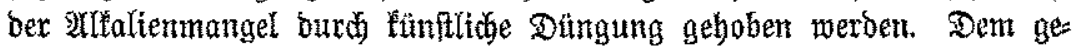
naurexen Etenogramm von ben jebenfalle bodinterefjanten Expojé wirb wobl mander Teilnebmer bex Serfanmlung mit Spanmung entgegen: 
Fehen; zweifelsobne wiro auc biefe neue "Jrofehre" (bies Mont jelbịt=

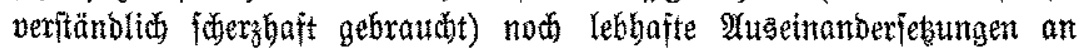

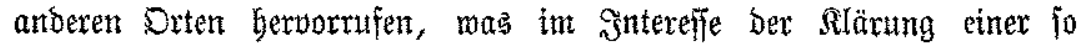
midbtigen Frage mur wïnifieneswert fein lann.

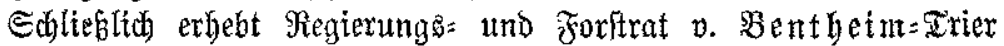

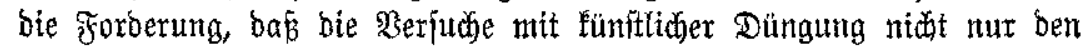

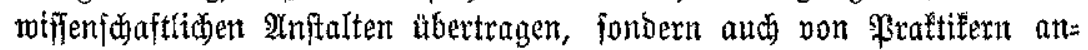
geitellt werben follen; am Roftenpunft weroe bie Düngungsifrage mobl

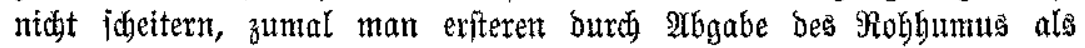
Streumaterial, namentliơ in Motjahten, verringern tönne.

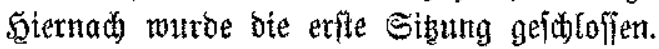

Madimittags wurbe ber berillynten Balhalla bei Donaliftauff, bem

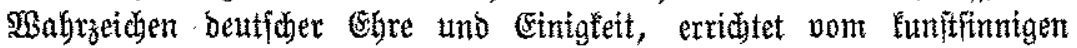

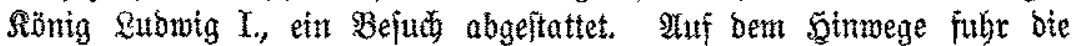

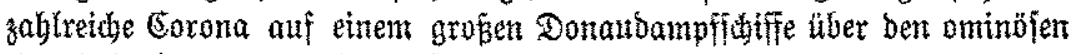
Strubel abwärts bis Donalitauff.

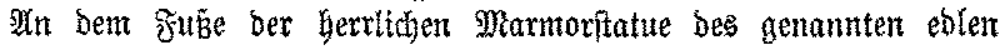
und "teuticheften" Fürften legte Der Sereinsvorfigende nach einer weibe=

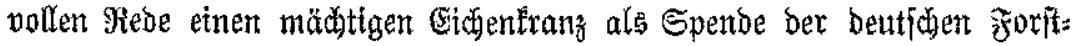
männer nieder.

Das für Dienstag arbeno geplante Ģartenfeft verregnete leiber, ob:

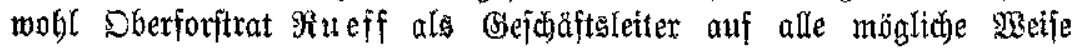

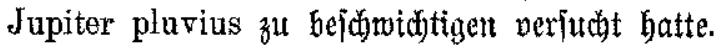

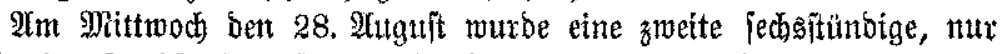

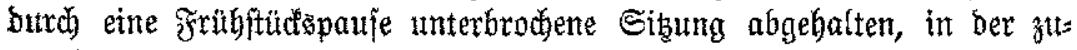

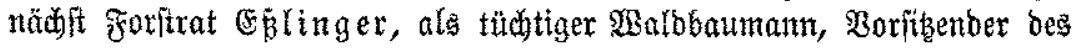

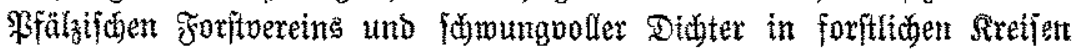
weit befant, bas Thema II einleite:

,Berubt in Femell flaguerfabren jowie in ber Rom bination besfelben mit bem Saumialagerfahren bas

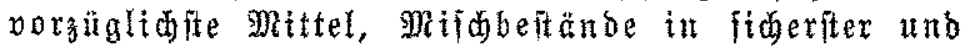
vollfommenfter Beife zu erziegen?"

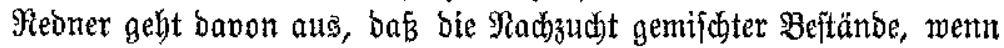
irgend möglid bas zu exftrebende Wirtiajaftsziel jein müffe und befiniert bas biejem $3^{\text {wedfe }}$ geredgt werbente Femelichlagnerfabren, wie es in

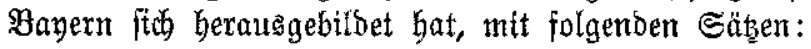

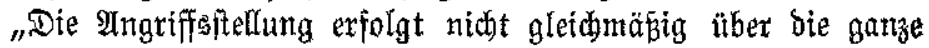
Fläche bes zut verjüngenden Beftandes, fonbern auf befduänfter Fłäche an mehreren Stelfen zugleich mit Belafiung gefoloffener

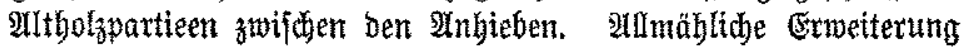




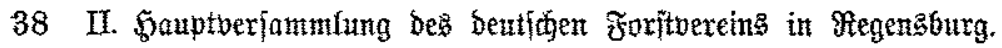

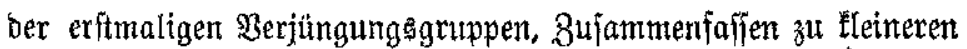

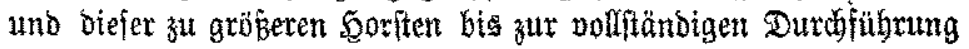
Der Beriüngung. ${ }^{.}$

Dieje erfolgt fomit unter \$ienthung eintger Samentahre, während bas fogenannte Dunkelfoblaguerfahren, wie es uriprünglid vornehnlitid für bte Buthe ausgebildet unb gelehnt wurbe, ein Samenjabr auf

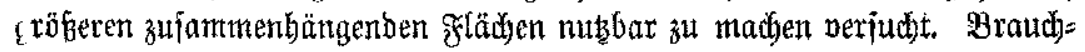
barer Sormudbs wirb in bie Berjî̉ngung einllezagen.

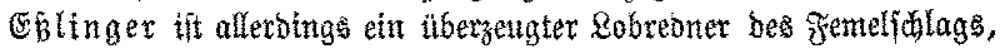

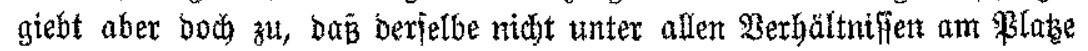

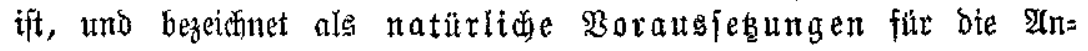
wenoung bes Berfahrens:

1. Mineralijob fräftigen unb frifben, Den Sctatthölżern genügenden Bober.

2. Bę̧üglich bes Terrains \$lateau's, (5benen, Mittelgebirge mit

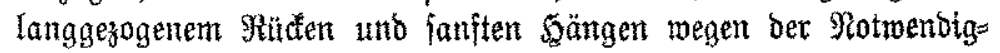

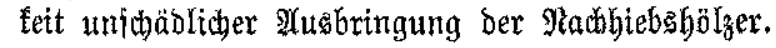

Sturmgefährbete Ragen finb für jenes wenig geeignet.

Meferent befpridit nun biejenigen Beriängungemethoden, welde unter

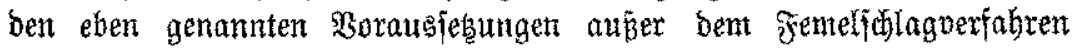

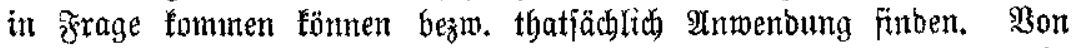
ben reinen \$ुlenterf̣ormen fiebt ex bierbei ab, weil bteje im Grobbetriebe

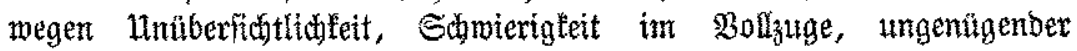

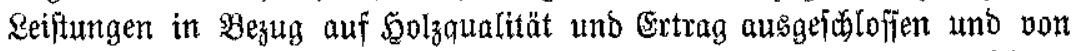

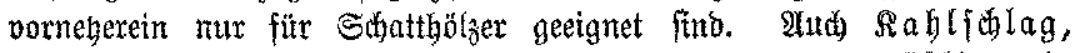

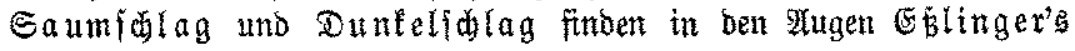
wentig Bnabe; über ben eriten jagt er:

„gahlidalag mit fünitlther Bejtanosbegrütrourg auf fabler

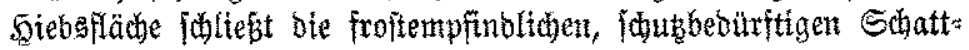

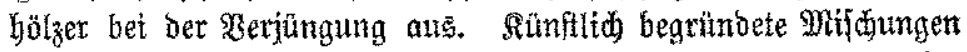

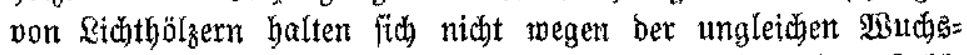

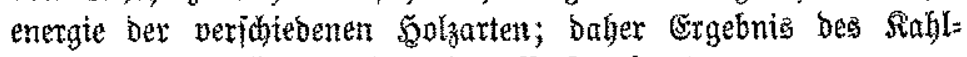

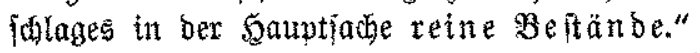

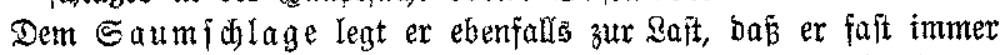
zu reinen Beftanbesfornen führe; jener tönte nur in nut bem Femelfolagoerfahren in Setradt fommen. Weiter äubert er fich itber ben Dunfelfchlag, bei bem bie erfite, über ben gröpten

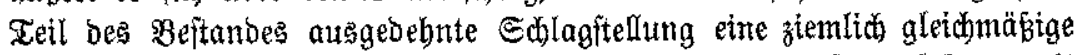

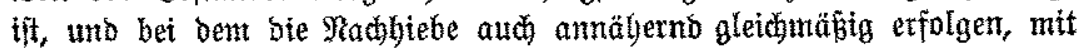
nadiftegenden Worten: 


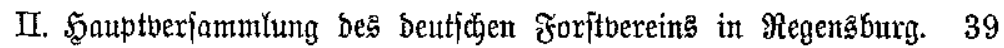

„Das Dunfelijdlagnerfahren ift ber Entftebung und Erhaltung von Mijømudge, fomie ber natürlidyen Berjüngung überhaupt wentger günftig als ber Femelifilag and zwar aus folgenten Gründen:

a) Die gleidumäzige Stellung bes Dunlelídlages gemäbrt bei gemifobter Beftoctung nur einer Solzart bas Spttmum

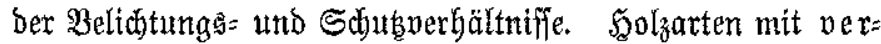
¡diebenen biologitchen 2tniprüchen bebingen eine räum=

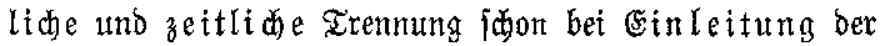

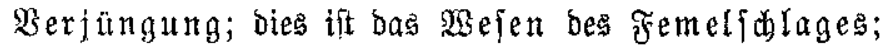
Shlagplfege fann es nicht eriegen.

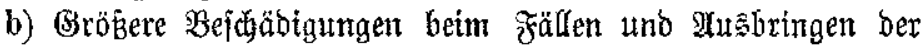
Nachbiebshölzer im Dunfelfulage.

c) Sonne und sind fönnen in bem gleid ntäbig gelichteten Dunfelijhlag mehr einwirfen, baher öfter Bobenverangerung und unregelmǟige Streuzerjeţung als in bem nur örtlid geöffnetent remelichlage.

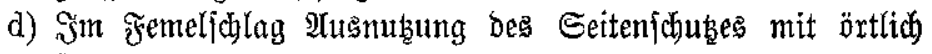
ftärferer \&ichtung ber $2 \mathfrak{n}$ wudisgruppen.

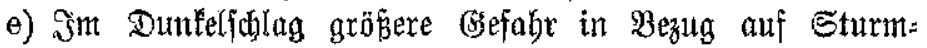

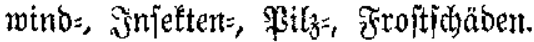

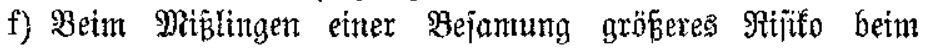

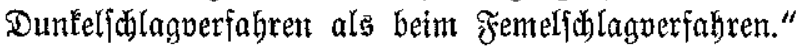

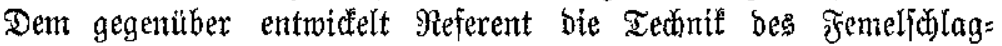
verfahrens, und zwar zunädyit bei lunterïtellung gefdolofiener uno in ber Sauptīache gleichalteriger $\mathfrak{B} e$ ftände. Şier geht ber Serjüngung bie leçete, fräftiger gefühtrte Durwforfitung als Borbereitungsfostag voraus, ferner Die Itnterfuchung etroiger Borwübje. Unthandibare werden ausgemultert; foldhe, weldhe eintwatben bürfen, namentlidy von Sdhatthölzerta wie $\mathfrak{Z} a n n e$,

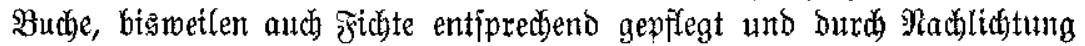
rinģum freigeitellt. Şn Den nidst mit Sorwudhs unteritandenen $\mathfrak{B} e=$

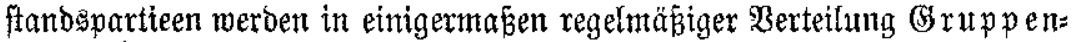
Giebe eingelegt, beren Eentra man mit Borliebe bafin verlegt, mo

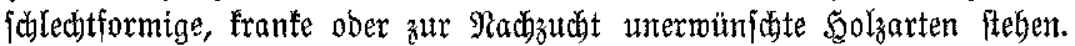

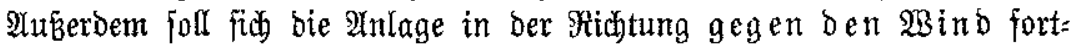
bewegen, jomie in ben bängen thunlidaft yon oben nach unten, um $\mathfrak{B}=$

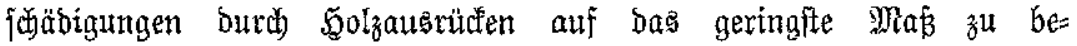
¡ọränteren.

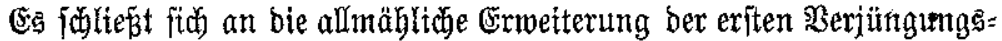

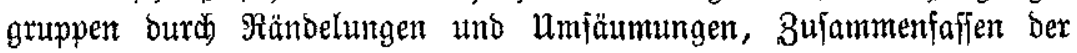




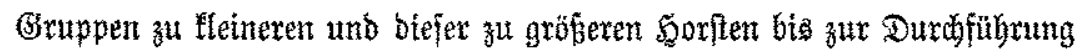

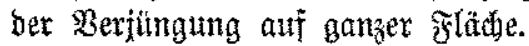

Unbejamt gebliebene Bwijhenjtreifen werben ohne langes Butwarten vom altheitanbe geräumt uno auegepflanzt; bagferbe geidetegt mit bem

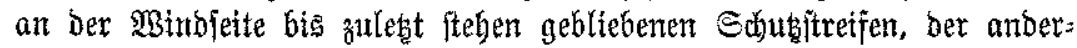
wärts auct Sturmbluc gentannt wiro.

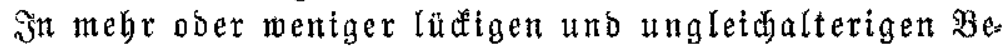

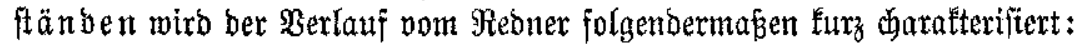

„Statt regelmäftg verteilter Şruppenbiebe, \$lenterbieb aụ älteres, ftärteres, abgängiges Material, Schaffung bolžleerex

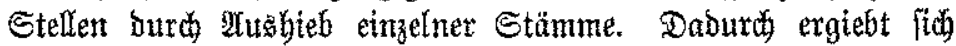

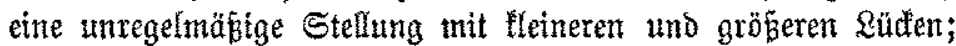

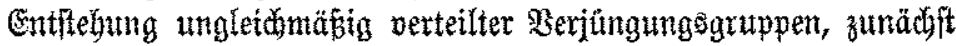
Arifedelung ber Sdattbölzer."

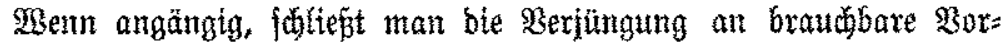

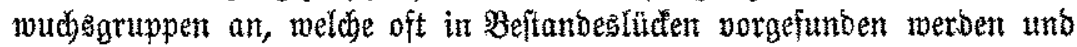

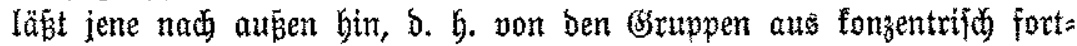

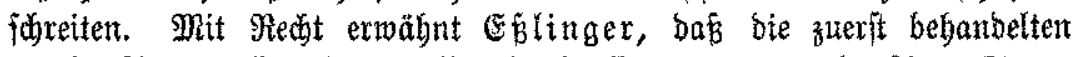

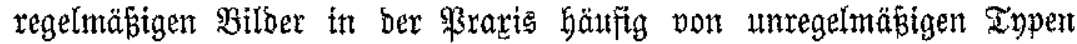

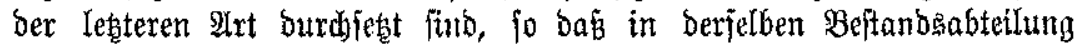
bals nad) ber eiten, bald nad ber anberen Midbififation gemirtidafitet werben mũ

Referent erfennt fernter an, bap verjhienene Standortes= und $B e:$

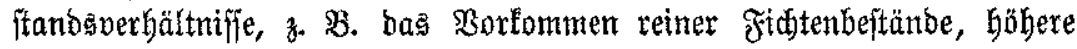

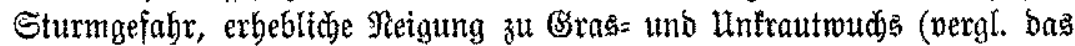

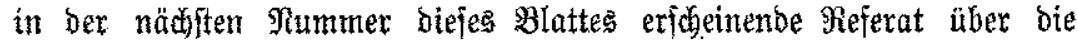

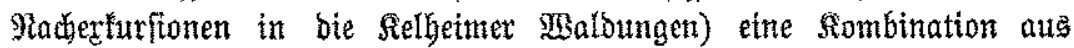

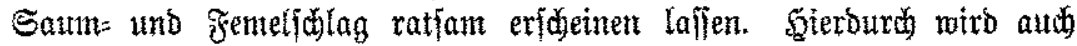

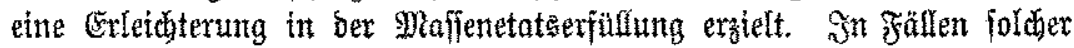
Urt werben vorwärs ber Edlagfront Gruppenbiebe gefithrt, in benen unter Umitänden auch in Thutterbeftande nidut vertretene Gajzer, z. $\mathfrak{B}$.

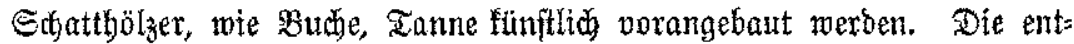
ftandenen Bormudisgrappen werben beint Boranjdreiten ber Saumbtebe in bie Beriütnung einbezogen.

Ejßlinget hebt nod) zwei wejentlthe Regeln bes Femelichlag:

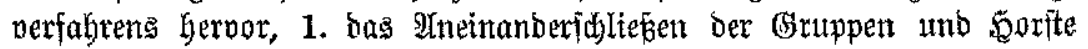
unter Bermeibung von Steilränbern, meil bieje fonft slitigleit ber

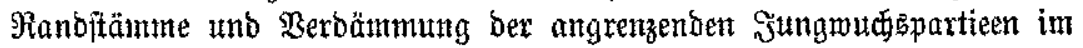
Gefolge haben. Es foll um ben Sern ber Soruppe ein um wentges

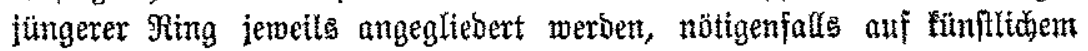


Sege. Zweitens beridftet ex, ba

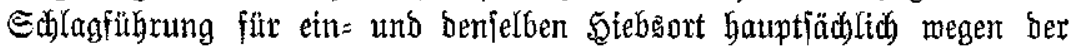

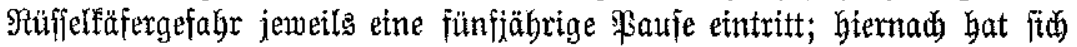
bie Schlagitellung zu ridjten.

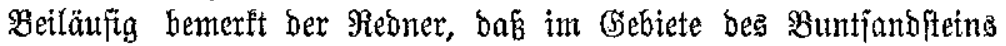

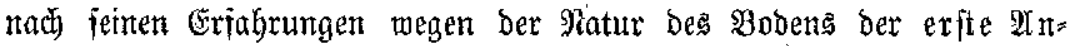

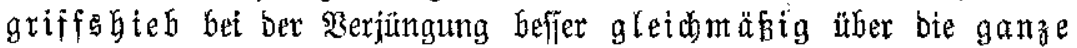

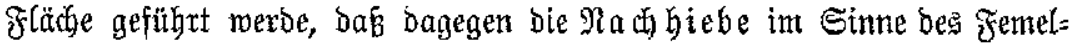
¡chlaguerfahrens gruppen= und Gorttweije einzulegen feien.

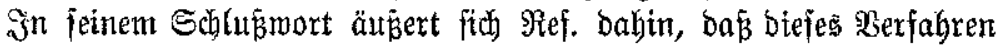
allerbing grob̉e Mannigfaltigkett bet der $\mathfrak{A}$ usführung zetge, indem ę fith bem jemeiligen sitht= und હdutshedürfniffe ber einzelnen 50 olzarten

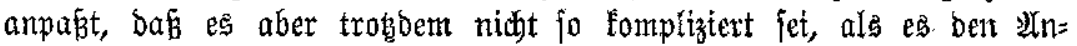
jusein habe. Seiner überzetgung nad) ift es vor allem vorzüglich ge= eignet, die Bobentraft zu berwahren und Mifabbejtände zut erziehen.

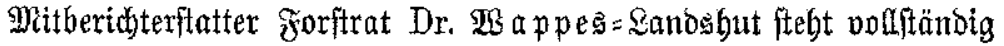

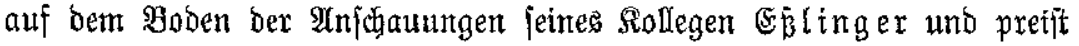
ben Femelfoblag int Gegenfabe zum Dunfelfohlage nantentlity megen ant=

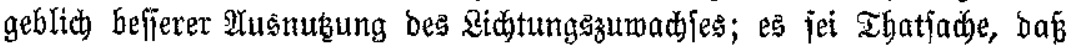

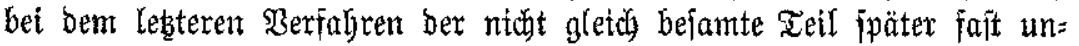
fähig zar Befanung werbe; audh führe bie geringere Bobenpflege unb

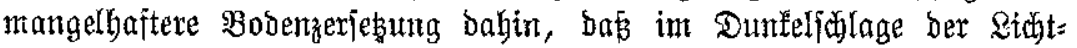

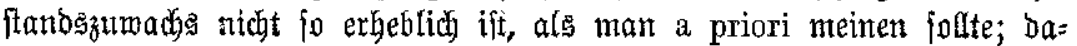

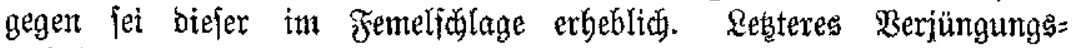

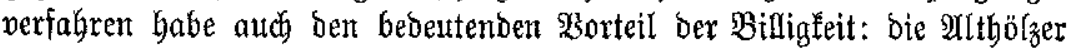
fönnen burab die nodb unbejamten Erellen befier anb obne Schaben zu madjen ausgerüft werben unb exfordern weit weniger fünftliche fiultur=

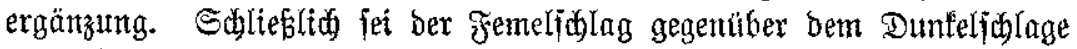
eine beweglichere Betriebsform, welde Berfangfanumgen und $\mathfrak{B e}=$

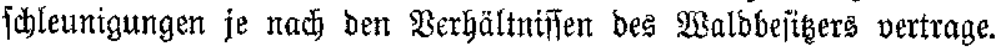

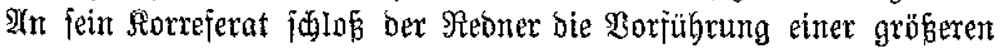

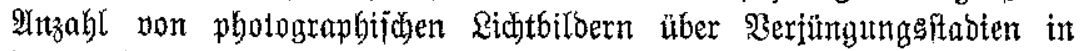

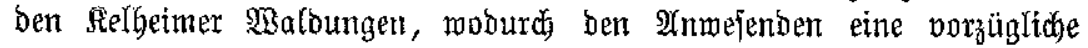

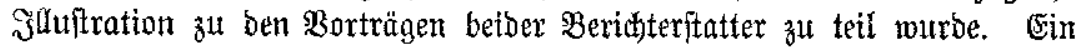
lebhaftes Bravo lohnte Gerrn $\mathfrak{B a p p e s}$ für bieje in ben Sibungen bes

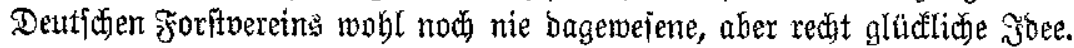

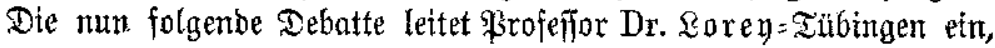
inbem ex bas won ben Borrebnern auf ben Femelidhlag gefungene $20 b=$ lieb boch in etwas herabftimmt; tener jei burchaus nidgt überafl nötig,

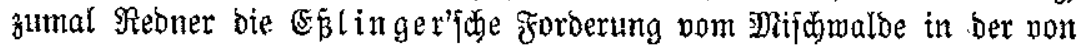




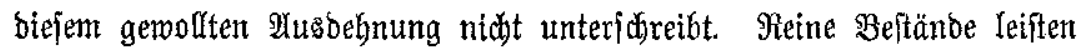

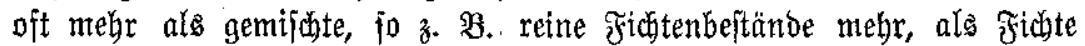

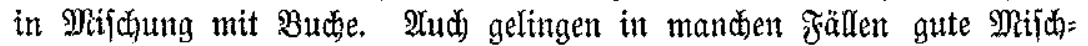

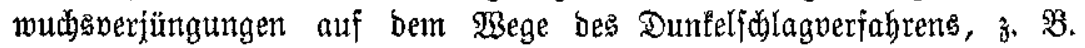

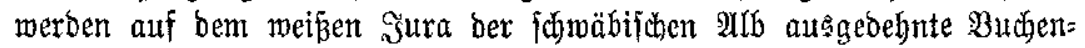

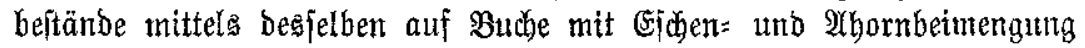

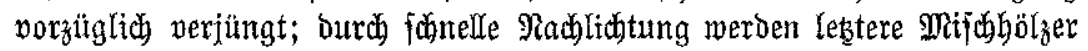
pouffert, wäbrend bie jonft verbämmende \$̧uthe zurürtbleibt.

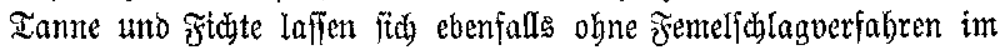

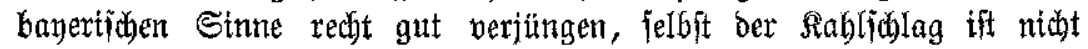
unbeoingt zu verwerfen.

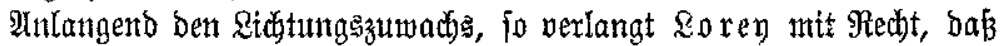
biejer vornehntlich burdh geeignteten Durdforitungsbetrteb bei Beiten an= zuitreben ift.

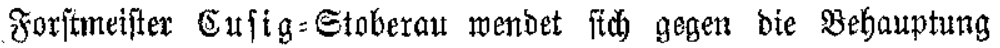

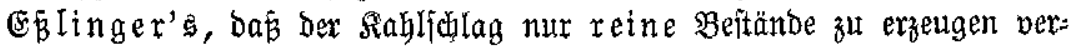
möge, inbem ex auf bie in jenem erzitelten Nadelholzberjüngungen in ben \$rovinzen Shlefien und Ditpreufen hinweift: Dort find Riefer unb Fidjte auf abwedbjelnoen Rablitreifen mit gutem Erfolge nadgezogen morden, wogeaen bas Femelfoblaguexfahren nicht immer zum ziele geführt hat.

Dberforftmeifter Dr. Borggreve = SBiesbaben erflärt als grund=

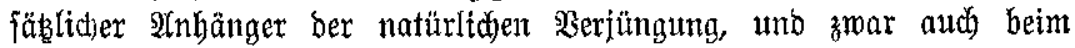

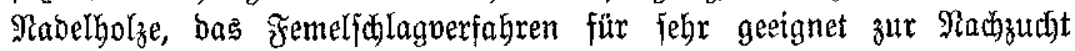
von Mijubeständen, bevorzugt bies jeboch nad) ber alter, von $\mathfrak{E a r l}$ Seyer vorgefdlagenen Methode Der mehr gleichmä żigen Schlagführumg.

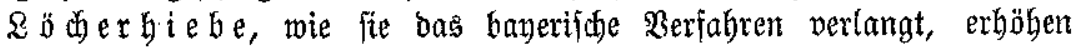
feines Eradtens bie Sturngefahr. Diejer Befüriftung giebt auch Dber= forftrat $\Re$ en $\tilde{\beta}=\mathfrak{M a ̈ h}$ rif Denlen begt, Dás fich aus Dem Femelichla g betriebe im zweiten umtriebe ber reine Femel= Doer \$glenterbetrieb entwidfeln wirb.

Den verigiedenen Sinmänden ber Borreoner gegenüber verteibigt Mintifterialrat v. 5 guber mit Märme jeinen Stebling, bas bayerifide

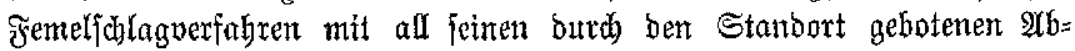
änderungen. Derfelbe jei der freiefte Betrieb, ber auf ber fleinen Fläble verichiedene Formen, auth ben ßablfhlag zulaffe. Da mo bie Sturmgefahr erheblicher ift, wirs zu ber Sombination mit bem Saum= ¡đlagverfahren gegriffen: jubrigens finb bie Windfälle feit Cinfübrung

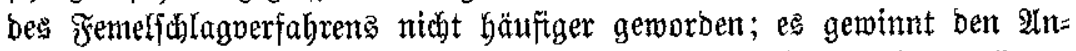

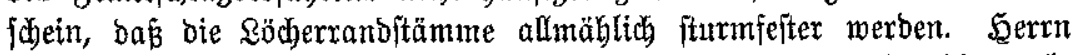

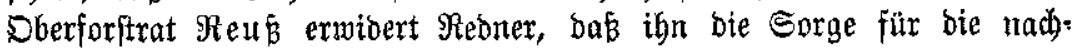


folgenben Untriebe falt laffe, wenn ex żmäefft einmal lebensfräftige Beftände auf gut erhaltenem Boben uno mit 2 ltersunteridieden von

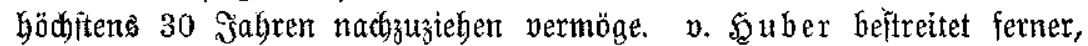

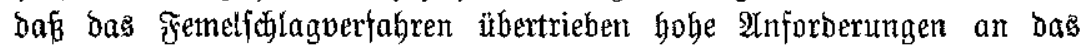
Berional ftellt und behauptet in biejer Finfitht nicht ohne berechtigten Stolz: „\$Bir in Bayern tönnen ung biejes Berfahren Leiften, benn wir

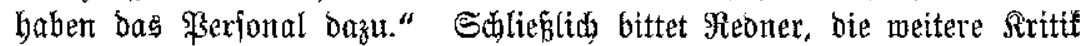
über jente auf bie Erfurfitonen zu veriparen, auf Denen bie Etgenart und ote Borzüge beşfelben flar in bie 2 tugen treten würbent.

Es folgten nunmebr die alläbrlid jublichen Mitteilungen über Serjuche, Beobactungen, Grfabrungen uno beachtensmerte :

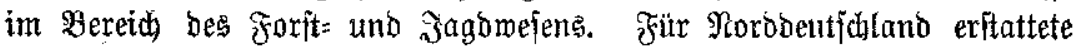

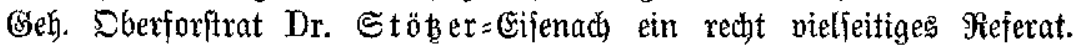

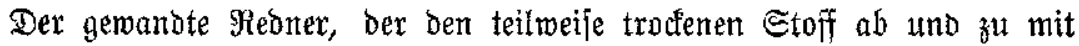
einer Rauge jalzigen WBizes anfeuthtet unb belebt, ergebt fich zunäbjit über bie umfangreichen Soblantbauffortungen in ben $\mathfrak{F r n v i n z e n ~ F a n n o v e r ~}$ und Schles̄wig: Şolittein. MUtein in ber eriteren fino in ben lesten 20 Sabren etwa 17000 ha in Seítand gebradyt moriden, wobei Der

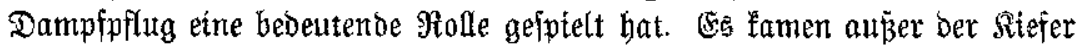

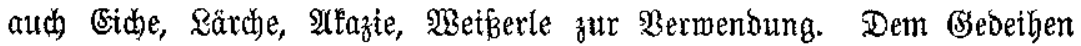
der Silturen murde teilweije mit fünitlidider Ditngung, u. a. auth mit Mergelbeigabe nadgebolfen.

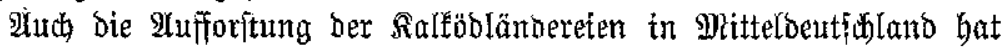

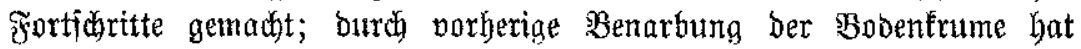

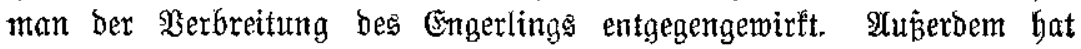
Gritnoüngung mit Reguminojen gute Dienite geleiftet; ebenjo bie 2 Un=

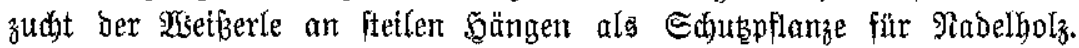

Dr. Stöber berichtet weiter über günftige Erfahrungen mit fünjt=

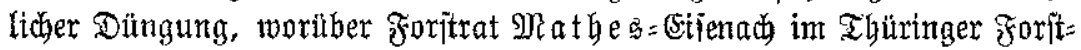
verein etren ausfulihrltthen Bortrag gehalten hat, uno exwähnt noch ins: bejonbere bie Exfolge bes Oberforitmeifters v. $\mathfrak{B a r e n b o r f f}=$ Stettin mit

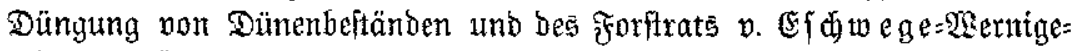

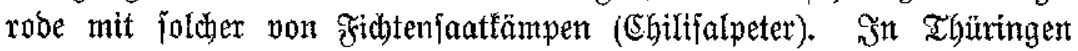

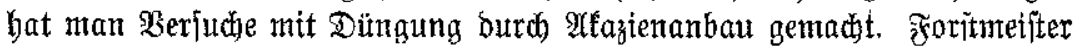

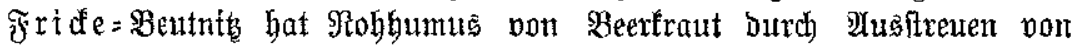
Rainit z̧um albfterben gebracht; anberwärts hat man fith bierzı bes 2 Am: montal=Superphosphates bebient. Der etwas reflamebaften Bebandung ber forftfichen Düngungsfrage burd Dr. Biersberg mibmet Rebner etrige farfátifiche semerfungen.

Er berichtet fermer über bie gegen bie Shütte getroffenen Máa: 


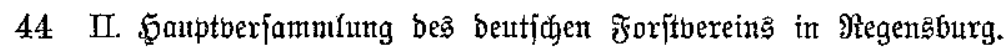

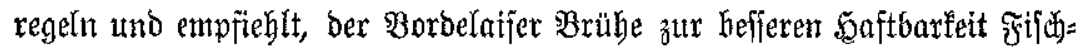
thran beižnnengen; er erwähnt auth, báz Düngung ber Schütte entgegen= wirft, und bas fräftige, verjafulte \$fflanzen biefem übel beffer wiber= ftehent.

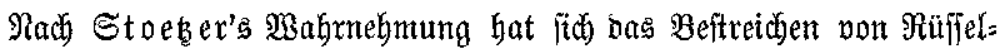

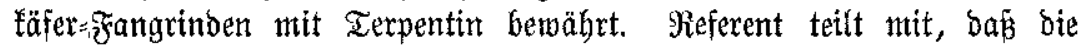

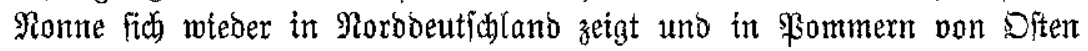

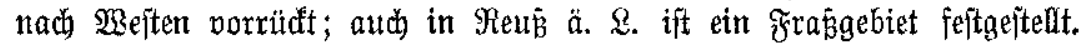

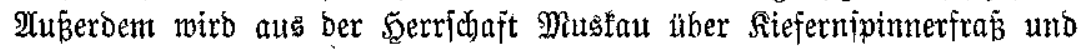

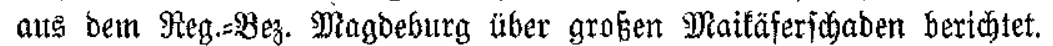

In Witteldeutjaland und am Sarz find bie Stürme wieber ver: berblich gemorden; auth murben verheerenbe Spätfröfte beobachtet, weldyent z. S. Die Douglag: und bie Sitfaficte zum Spfer fielen.

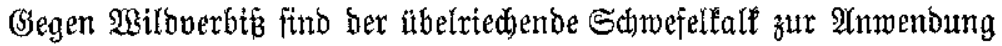
gelangt, ferner bas Bemidfeln mit $\mathfrak{B} e r g$, bas Beftrethen mit der

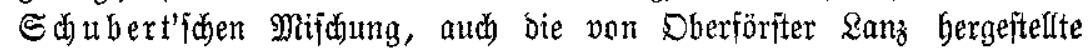

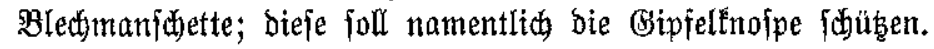

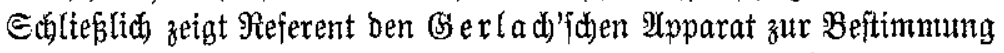

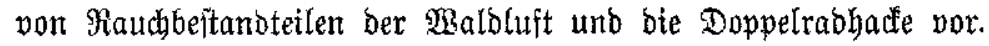

Dberforitrat \$rofefior Siefert:Sarlsube madt eingebenoe Mit= teilungen über bas 2luftreten non Drtiteinhíloungen, meldye häuftger finb, als man feither angenommen bat und insbefondere auch unter burch)=

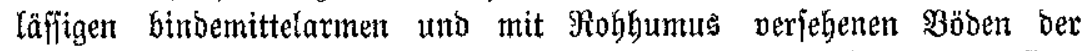
Buntfanditein=, Diluvial= und Grantiformation feitgeitellt worben find. Rebner warnt auf jolchen Böben vor Rahlhieben und befürwortet, von

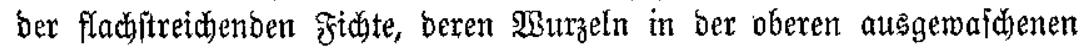

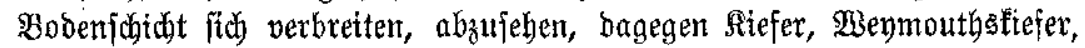

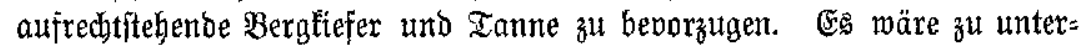

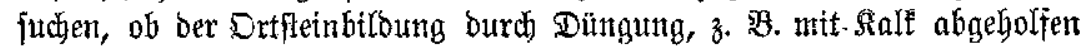
mernen fönute.

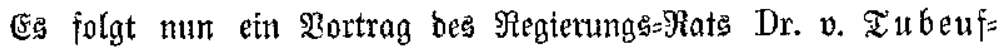
Berlin über bie Berbreitung des \$Benmoutbgftefernblafenroftes, welder in 107 Forttrevieren Deutichlands und in $86^{\circ}$ Privatwalbungen nact)=

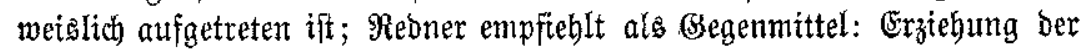

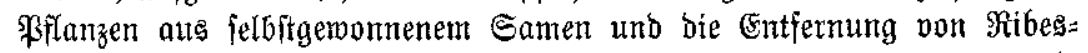

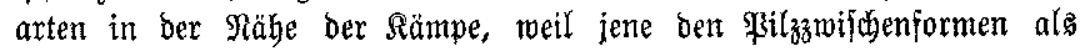
Reimbett bienen.

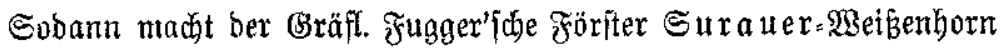

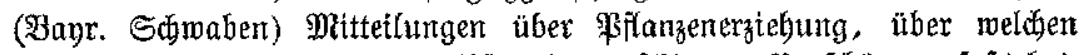

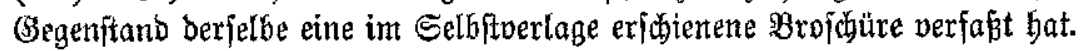




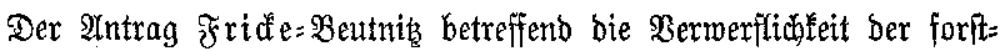

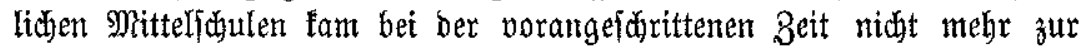
Erörterung, foll bagegen als bejonberes Ihema ber näbjtä̈brigen $\mathfrak{L} a g u n g$ zu Reipzig behanbelt werden.

Buguterleşt möge noch Erwähnung finden, Dás Dberforitmeifter Dr. Borggreve ber Berjammlung ein Hleines 2 Angebinbe madhte, nänlidid

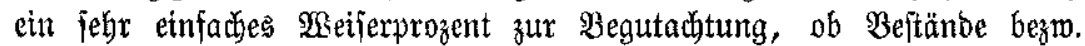
einzelne Stämme nod angemeffen rentieren. (Ex behauptet, dab nod eine $\mathfrak{B}$ erzinjung von 3 pert. vorliegt, wenn 6 Sabrringe auf ben Eenti= meter Deg jüngiten Sgolźtörpers gehen, eine joldye von $4^{1 / 2}$ pet. bet 5 , von 5p pet. bei 4 und von 6 pđt. bei 3 sabrringent. Sorggreve hat veriproben, im Berfammlungsberidite Den Bemeis zu erbringen.

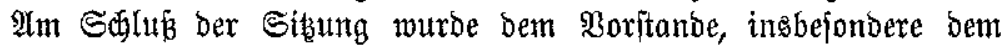

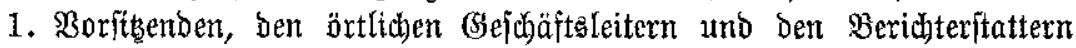
Der gebührende Danf abgeftattet. Das Nachmittage fth anjobliebende Feîtmabl verlief wie immer beiter und war burd bie obligate Serie von Ioaften gewürzt. Mix wollen insbejondere den auf ben Frinzregenten

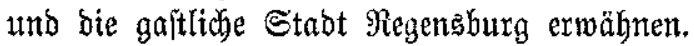

$\mathrm{X}$.

\section{Das 2 bffecten von Kreisfurven.}

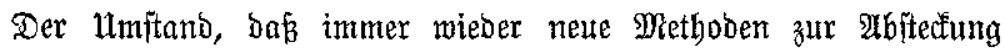
von Sreisfurben aufgejucht werben, legt bie : Sernutung nabe, baj ein

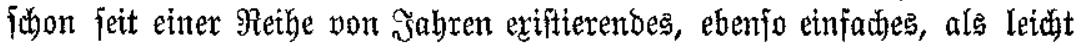

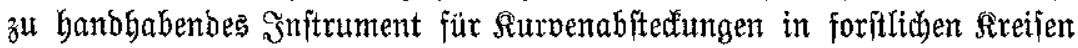
nur fefr wenig befannt ift. Es i ift bies bie \$rismentrontmel, fomitruiert

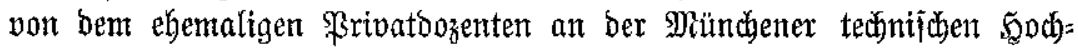
idulle, Dr. Dediert. Das Jnitrument bejteht aus zwet vertifal über einander angeoroneten \$rismen, von benen bas eine fejtítegt, wäbrento

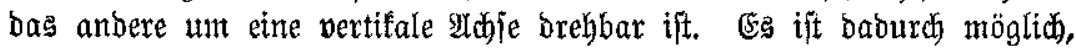

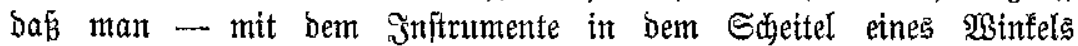

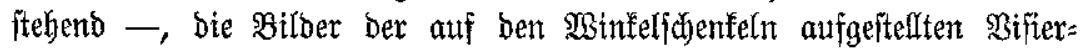

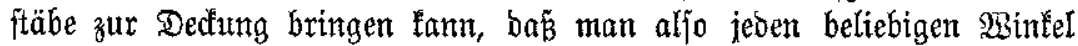

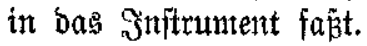

Die $\mathfrak{A n w e n b u r g}$ bes Sinitruments berubt auf bem genmetrijben

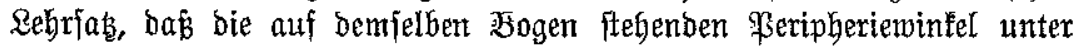
einander gleid) und gleich find bem zugehörigen Sehnentangentenminfel (bem. WSinfel, welchen bie Tangente im Berührungspunfte mit ber ben Bogen abfdnetbenden Sebne bildet). Bur 2rbittechung ber ßurve ftellt 